\title{
Gestão e plano de contingência do Serviço de Imunoalergologia do Centro Hospitalar e Universitário de S. João no contexto da pandemia por COVID-19
}

\section{Managing an Allergy and Clinical Immunology Department in a portuguese reference COVID-19 hospital during pandemic contingency}

Rev Port Imunoalergologia $2020 ; 28$ (3): I6I-I7I

Leonor Carneiro-Leão, Luís Amaral, Alice Coimbra, José Luís Plácido

Serviço de Imunoalergologia - Centro Hospitalar de São João, Porto

Todos os autores contribuíram igualmente para este trabalho

\section{RESUMO}

A pandemia por SARS-CoV-2 disseminou-se rapidamente por todo o Mundo, infetando um enorme número de pessoas e causando inúmeros óbitos. À semelhança de muitos outros países, Portugal declarou estado de emergência nacional e aplicou medidas de confinamento rapidamente após a declaração de pandemia pela Organização Mundial de Saúde. Estas medidas resultaram em alterações dramáticas e inesperadas à atividade clínica, às quais tivemos de nos adaptar.

Foi de imediato desenhado um plano de contingência, que incluiu estratégias inovadoras, de modo a garantir não só a prestação de cuidados de saúde aos nossos doentes alérgicos, mas também a segurança dos profissionais de saúde. Para além disso, era fundamental ter em consideração a participação intensa da maioria dos médicos do Serviço de Imunoalergologia em atividades relacionadas com a COVID-19. Este plano foi particularmente difícil de elaborar, 
dado que, à data, não existiam quaisquer recomendações ou guidelines. Consideramos que a partilha desta experiência com outros serviços e unidades de imunoalergologia poderá facilitar a adaptação de todos no caso de uma futura vaga de COVID-19 ou outra pandemia.

Palavras-chave: Doenças alérgicas, COVID-19, imunoalergologia, plano de contingência.

\section{ABSTRACT}

The SARS-CoV-2 pandemic spread worldwide in a short period causing an extremely high number of infected people and numerous fatalities. Portugal as many other countries declared a State of National Emergency and applied quarantine measures soon after the WHO declaration of pandemic. As result we had to deal with dramatic and unexpected changes affecting our clinical activity on an unprecedented scale. A Contingency Plan was immediately planned, comprising new approaches and strategies, to assure the health care needs of our allergic patients and the safety of both health care providers and patients. Additionally, we needed to consider the intensive participation of most of the doctors and nurses of our Department in COVID-19 related activities. The Contingency Plan was also particularly difficult to elaborate since no recommendations or guidelines were available at that time. We believe that sharing this experience with other Allergy and Clinical Immunology Departments may prove to be useful in case of a second wave of COVID-19 infection or another future pandemic.

Key-words: Allergic diseases, COVID-19, immunoallergology, contingency plan.

\section{INTRODUÇÃO}

A 31 de dezembro de 2019, a China reportou à Organização Mundial da Saúde (OMS) um cluster de pneumonia de etiologia desconhecida em trabalhadores e frequentadores de um mercado de peixe, mariscos vivos e aves na cidade de Wuhan, província de Hubei. A 9 de janeiro de 2020 as autoridades chinesas identificaram um novo vírus da família dos coronavírus (2019-nCoV, posteriormente rebatizado SARS-CoV-2) como agente causador da doença'.

O número de casos aumentou muito rapidamente por todo o mundo durante as semanas seguintes, tendo sido reconhecida a sua elevada contagiosidade e potencial patogénico ${ }^{2}$. A OMS declarou a 30 de janeiro de 2020 a doença pelo novo coronavírus como uma emergência de saúde pública de âmbito internacional e, como pandemia a II de março de $2020^{3}$.

Em Portugal, os primeiros casos de infeção foram confirmados a 2 de março de 2020, no Porto. Um dos casos tratava-se de um médico de 60 anos que tinha passado férias no Norte Itália e o outro era um trabalhador de 33 anos que tinha regressado ao país proveniente de Valência. O primeiro óbito por COVID-19, um doente de 80 anos, foi registado a 16 de março no Centro Hospitalar Universitário Lisboa Norte. Três dias depois, a 18 de março, foi declarado o estado de emergência e decretadas medidas de quarentena que vigoraram até ao dia 2 de maio (Figura I). Até 2 de maio foram registados em Portugal 25190 doentes infetados e 1023 óbitos por COVID-194.

O Centro Hospitalar Universitário de S. João (CHUSJ) foi ativado pela Direção-Geral de Saúde (DGS) como 


\section{GESTÃO E PLANO DE CONTINGÊNCIA DO SERVIÇO DE IMUNOALERGOLOGIA \\ DO CENTRO HOSPITALAR E UNIVERSITÁRIO DE S. JOÃO \\ NO CONTEXTO DA PANDEMIA POR COVID-19 / PÁGINA EDUCACIONAL}

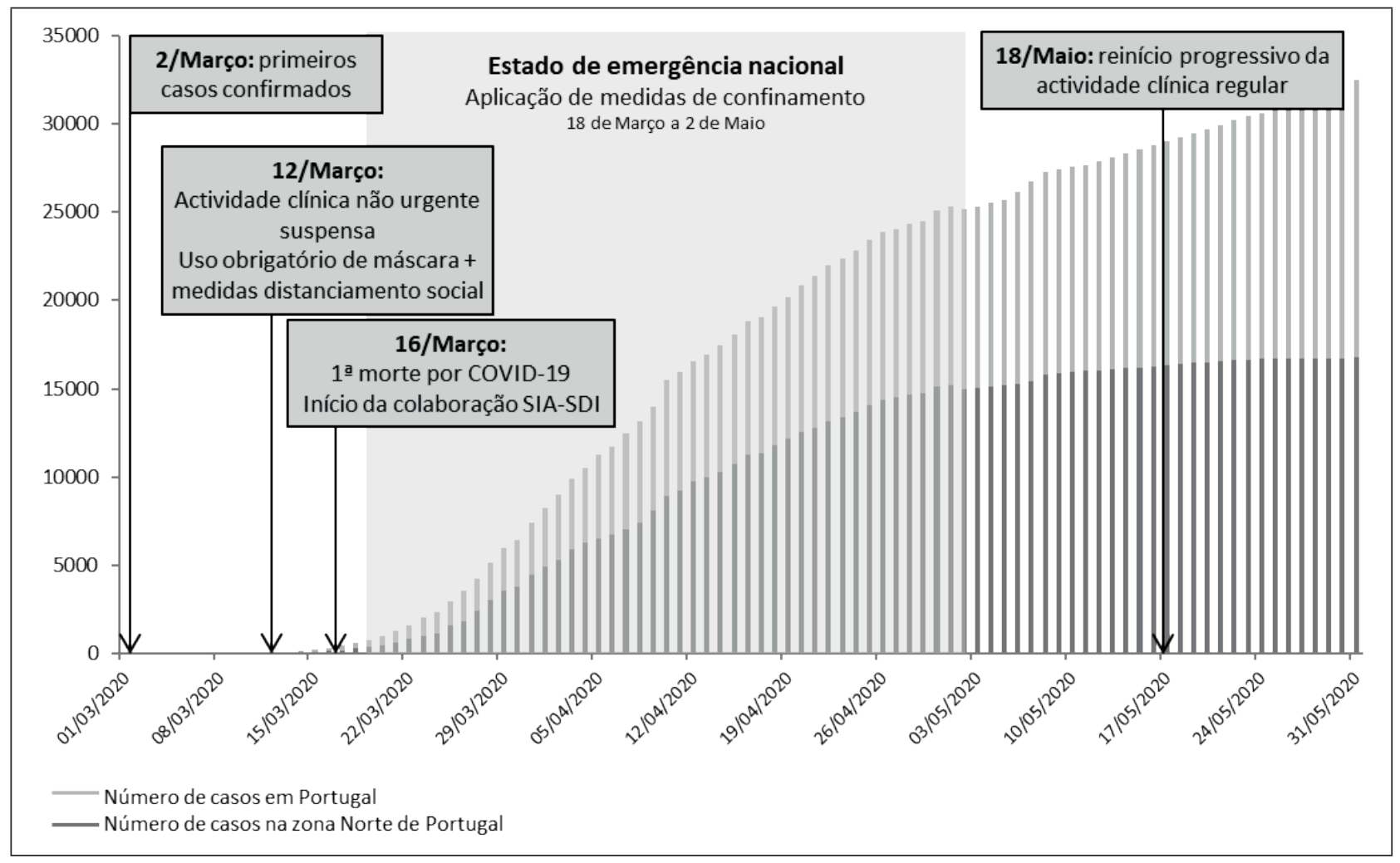

SIA: Serviço de Imunoalergologia; SDI: Serviço de Doenças Infeciosas.

Figura I. Evolução da pandemia COVID-19 em Portugal e timeline das medidas de confinamento e atividade clínica do Serviço de Imunoalergologia.

hospital de referência da região Norte para a COVID-19 em janeiro de 2020. A região Norte, nomeadamente o distrito do Porto, foi o epicentro inicial da COVID-19 no nosso país. A 12 de março de 2020 e por indicação do conselho de administração e da direção clínica, toda a atividade clínica e cirúrgica programada do CHUSJ foi suspensa, com os seus recursos redirecionados para atividades relacionadas com a COVID-19.

Durante o período compreendido entre 12 de março e 2 de maio, foram implementadas medidas muito restritivas relativas à entrada e circulação de pessoas no CHUSJ, e de distanciamento social, bem como o uso obrigatório de máscara para os profissionais de saúde e utentes. $\mathrm{O}$ acesso ao CHUSJ passou a ser possível apenas por duas entradas, tendo sido criados check-points nestas zonas para deteção de utentes ou profissionais de saúde com sintomas suspeitos e/ou febre, em linha com as recomendações do Centro Europeu de Controlo de Doenças, OMS e DGS²,4,5.

Foi disponibilizado o acesso remoto aos profissionais de saúde a aplicações informáticas para realização de teletrabalho e os telefones hospitalares foram desbloqueados à rede externa. Durante este período, o CHUSJ acompanhou aproximadamente 2000 doentes infetados, contabilizou 500 internamentos e 110 óbitos por COVID-19.

Com o final do estado de emergência, as atividades clínicas e cirúrgicas programadas foram retomadas gradualmente pelos vários serviços. O Serviço de Imunoalergologia reiniciou as suas atividades a partir do dia 18 de maio (Figura I, Quadro I).

Estas determinações tiveram um impacto tremendo na atividade do Serviço de Imunoalergologia do CHUSJ. A atividade clínica presencial e não urgente foi quase 
Quadro I. Plano de ação relativo às diversas atividades do Serviço de Imunoalergologia e o número de atividades realizadas/ /programadas durante o período compreendido entre 12 de março e 18 de maio de 2020.

\begin{tabular}{|c|c|c|c|}
\hline $\begin{array}{l}\text { Consultas do Serviço } \\
\text { de Imunoalergologia }\end{array}$ & Plano de ação & $\begin{array}{l}\text { Presenciais/ } \\
\text { /teleconsulta/ } \\
\text { /programadas }\end{array}$ & Retoma da atividade \\
\hline \multicolumn{4}{|c|}{ Consultas médicas } \\
\hline I. as Consultas & $\begin{array}{l}\text { Canceladas até ao fim de abril, } \\
\text { exceto em situações graves/ } \\
\text { /urgentes }\end{array}$ & $26 / 189 / 600$ & $\begin{array}{l}\text { Retomadas em maio, com } \\
\text { duplicação do n. }{ }^{\circ} \text { de } 1 .{ }^{\text {as }} \text { consultas } \\
\text { para recuperar desmarcadas }\end{array}$ \\
\hline Consultas subsequentes & $\begin{array}{l}\text { Conversão em teleconsultas } \\
\text { sempre que possível.A decisão foi } \\
\text { baseada na gravidade clínica }\end{array}$ & $43 / 1838 / 1900$ & $\begin{array}{l}\text { Maioritariamente não } \\
\text { presenciais até meados } \\
\text { de junho }\end{array}$ \\
\hline $\begin{array}{l}\text { Outras atividades } \\
\text { do Serviço de } \\
\text { Imunoalergologia }\end{array}$ & Plano de ação & $\begin{array}{l}\text { Atividades } \\
\text { realizadas/ } \\
\text { /programadas }\end{array}$ & Retoma da atividade \\
\hline \multicolumn{4}{|c|}{ Imunoterapia com alergénios } \\
\hline Iniciação de aeroalergénios & Suspensa & $0 / 20$ & Retomada como habitual. \\
\hline Manutenção de aeroalergénios & $\begin{array}{l}\text { Suspensa. Os doentes foram } \\
\text { incentivados a manter tratamento } \\
\text { nos cuidados de saúde primários }\end{array}$ & $17 / 604$ & $\begin{array}{l}\text { Retomada com intervalos de } \\
\text { tempo mais longos (para evitar } \\
\text { sobrelotação das salas de espera) }\end{array}$ \\
\hline $\begin{array}{l}\text { Iniciação com veneno de } \\
\text { himenópteros }\end{array}$ & Suspensa & $0 / 5$ & Retomada como habitual \\
\hline $\begin{array}{l}\text { Manutenção com veneno de } \\
\text { himenópteros }\end{array}$ & $\begin{array}{l}\text { Mantida após informação ao } \\
\text { doente dos riscos/benefícios }\end{array}$ & $60 / 65$ & Nunca suspensas \\
\hline \multicolumn{4}{|c|}{ Alergia alimentar } \\
\hline Testes em picada-picada & Suspensos & $0 / 75$ & Retomada como habitual \\
\hline Provas de provocação oral & Suspensos & $0 / 62$ & Retomada como habitual \\
\hline Indução de tolerância oral & $\begin{array}{l}\text { Iniciações foram suspensas. } \\
\text { Mantidos os tratamentos em } \\
\text { curso sem subida de dose }\end{array}$ & $0 / 4$ & Retomada como habitual \\
\hline \multicolumn{4}{|c|}{ Alergia a fármacos } \\
\hline $\begin{array}{l}\text { Testes cutâneos/ Provas de } \\
\text { provocação }\end{array}$ & Suspensos & $0 / 188$ & Retomada como habitual \\
\hline Dessensibilizações & Mantida para antineoplásicos & $16 / 16$ & Nunca suspensas \\
\hline
\end{tabular}

totalmente suspensa. Os médicos do serviço foram chamados a participar nas atividades clínicas relacionadas com a COVID-19, designadamente o seguimento por teleconsulta de doentes infetados, atividades nas enfermarias de doentes internados com COVID-19 e turnos de urgência na avaliação de doentes com suspeita de COVID-19. Neste período, o espaço físico da Consulta Externa de Imunoalergologia no Centro de Ambulatório Médico foi alocado na sua quase totalidade ao Serviço de Saúde Ocupacional do CHUSJ, para rastreio e seguimento dos profissionais de saúde com COVID-19.

Estas dramáticas e inesperadas alterações colocaram-nos perante desafios difíceis e a necessidade de tomar rápidas decisões. Foi-nos requerida a elaboração de um plano de contingência que assegurasse a segurança dos profissionais de saúde e dos doentes, a resposta às solicitações relacionadas com a COVID-19 e o seguimento dos doentes com patologia imunoalergológica. Esta situa- 


\section{GESTÃO E PLANO DE CONTINGÊNCIA DO SERVIÇO DE IMUNOALERGOLOGIA \\ DO CENTRO HOSPITALAR E UNIVERSITÁRIO DE S. JOÃO \\ NO CONTEXTO DA PANDEMIA POR COVID-I9 / PÁGINA EDUCACIONAL}

(continuação)

\begin{tabular}{|c|c|c|c|}
\hline $\begin{array}{l}\text { Consultas do Serviço } \\
\text { de Imunoalergologia }\end{array}$ & Plano de ação & $\begin{array}{l}\text { Presenciais/ } \\
\text { Iteleconsulta/ } \\
\text { /programadas }\end{array}$ & Retoma da atividade \\
\hline \multicolumn{4}{|c|}{ Provas de função respiratória } \\
\hline $\begin{array}{l}\text { Espirometrias com/sem } \\
\text { broncodilatação }\end{array}$ & \multirow{4}{*}{ Suspensas } & $0 / 606$ & \multirow{4}{*}{$\begin{array}{l}\text { Restritas a } I / 3 \text { da atividade } \\
\text { habitual, com recurso a EPI } \\
\text { e reforço das medidas de } \\
\text { desinfeção }\end{array}$} \\
\hline Fração exalada de óxido nítrico & & $0 / 437$ & \\
\hline $\begin{array}{l}\text { Broncoprovocação com } \\
\text { metacolina }\end{array}$ & & $0 / 25$ & \\
\hline Oscilometria de impulso & & $0 / 20$ & \\
\hline $\begin{array}{l}\text { Testes cutâneos por picada } \\
\text { com aeroalergénios }\end{array}$ & Suspensos & $0 / 510$ & Retomada como habitual \\
\hline Terapêutica com biológicos & $\begin{array}{l}\text { Mantida. Conversão para } \\
\text { autoadministração sempre que } \\
\text { possível }\end{array}$ & $29 / 46 *$ & $\begin{array}{l}\text { Continuação do programa } \\
\text { de autoadministração de } \\
\text { biológicos }\end{array}$ \\
\hline \multicolumn{4}{|c|}{ Serviço de atendimento não programado } \\
\hline Consultas urgentes & \multicolumn{2}{|c|}{ Restritas a doentes com sintomas cutâneos } & Manutenção das restrições \\
\hline Consultas internas & \multicolumn{2}{|l|}{ Mantidas } & Nunca suspensas \\
\hline Apoio ao serviço de urgência & \multicolumn{2}{|l|}{ Mantido } & Nunca suspensa \\
\hline \multicolumn{4}{|c|}{ Equipa médica } \\
\hline \multirow{4}{*}{$\begin{array}{l}\text { Medidas de distanciamento } \\
\text { social }\end{array}$} & \multicolumn{2}{|c|}{$\begin{array}{l}\text { Reuniões e comunicações entre os profissionais de saúde } \\
\text { realizadas através de smartphones hospitalares, WhatsApp }{ }^{\circledR} \\
\text { ou correio eletrónico }\end{array}$} & $\begin{array}{l}\text { Retomadas, com medidas de } \\
\text { distanciamento social }\end{array}$ \\
\hline & \multicolumn{2}{|c|}{ Teleconsultas realizadas a partir do domicílio } & Suspensão do teletrabalho (junho) \\
\hline & \multicolumn{2}{|c|}{$\begin{array}{l}\text { Contacto com os doentes através de smartphones } \\
\text { hospitalares, WhatsApp }{ }^{\circledR} \text {, correio eletrónico }\end{array}$} & $\begin{array}{l}\text { Mantido o contacto com doentes } \\
\text { através de plataformas digitais }\end{array}$ \\
\hline & \multicolumn{2}{|l|}{ Prescrições eletrónicas } & Mantidas \\
\hline $\begin{array}{l}\text { Participação nas atividades } \\
\text { COVID-19 }\end{array}$ & \multicolumn{2}{|c|}{$\begin{array}{l}\text { Participação voluntária nas atividades relacionadas } \\
\text { com a COVID- 19, incluindo integração nas equipas de } \\
\text { internamento dedicadas, consulta e SU. Inclui a realização } \\
\text { de } 5255 \text { das } 1 \text { I } 090 \text { teleconsultas realizadas a doentes com } \\
\text { COVID- } 19 \text { sob tratamento domiciliário. }\end{array}$} & Terminada (junho) \\
\hline \multicolumn{4}{|c|}{ Atividade Científica e Formativa } \\
\hline Investigação & \multicolumn{2}{|c|}{$\begin{array}{l}\text { Suspensa nos casos em que havia contacto presencial. } \\
\text { Redirecionada para COVID-19 }\end{array}$} & $\begin{array}{l}\text { Retomados projetos de } \\
\text { investigação em IA e } \\
\text { continuação de projetos em } \\
\text { COVID-I9 }\end{array}$ \\
\hline \multirow{2}{*}{ Formação médica contínua } & \multicolumn{2}{|c|}{$\begin{array}{l}\text { Reuniões científicas foram suspensas. Discussão de casos } \\
\text { clínicos através de smartphones hospitalares ou WhatsApp }{ }^{\circledR}\end{array}$} & $\begin{array}{l}\text { Retomadas, com medidas de } \\
\text { distanciamento }\end{array}$ \\
\hline & \multicolumn{2}{|c|}{$\begin{array}{l}\text { Participação em reuniões científicas nacionais ou } \\
\text { internacionais em plataformas digitais }\end{array}$} & $\begin{array}{l}\text { Mantidas maioritariamente via } \\
\text { plataformas digitais }\end{array}$ \\
\hline $\begin{array}{l}\text { Estágios do internato } \\
\text { de formação específica }\end{array}$ & \multicolumn{2}{|l|}{ Suspensos } & Retomados \\
\hline
\end{tabular}

* n. doentes que aderiram ao programa de autoadministração no domicílio/total de doentes sob terapêutica com biológicos

¥ Inclui crianças em idade pré-escolar 
ção foi particularmente difícil de gerir numa fase inicial, em que não estavam ainda disponíveis quaisquer recomendações ou guidelines relativas à gestão dos serviços ou unidades de imunoalergologia durante a pandemia COVID-19. O primeiro artigo a mencionar algumas destas recomendações foi publicado on-line apenas no final de março por Codispoti et $a l^{6}$.

A existência de um plano de contingência nos serviços ou unidades de imunoalergologia é fundamental para resposta futura a situações de emergência, de que esta pandemia é um bom exemplo. Vimos assim dar a conhecer a forma como o Serviço de Imunoalergologia se reorganizou durante as oito semanas iniciais da pandemia (de 12 de março a 18 de maio), período em que toda a atividade clínica programada foi praticamente suspensa no CHUSJ. Consideramos que a partilha desta experiência com outros serviços ou unidades de imunoalergologia poderá vir a ser útil numa segunda vaga de infeção por COVID-19 ou outra futura pandemia.

\section{ATIVIDADE CLÍNICA}

\section{Consulta externa}

As primeiras consultas e as consultas subsequentes foram quase totalmente suspensas, com as subsequentes a serem maioritariamente convertidas em teleconsultas, geralmente realizadas pelos médicos a partir de suas casas, ou mais raramente a partir de um posto de trabalho no hospital.

Os doentes foram avisados por SMS de que a atividade em regime de consulta externa tinha sido genericamente suspensa. A 24 horas do dia da consulta recebiam novo SMS pedindo para não se deslocarem ao hospital e informando que a consulta seria realizada por via telefónica pelo seu imunoalergologista assistente. Em situações clínicas excecionais, por decisão médica, foi solicitado aos doentes, também por SMS, que se deslocassem ao hospital para serem observados presencialmente. Neste caso, as consultas foram agendadas em horários especí- ficos e com intervalos de tempo alargados, de forma a reduzir o número de doentes nas salas de espera e o seu tempo de permanência no hospital.

Durante o período compreendido entre 12 de março e 18 de maio foram realizadas cerca de 3200 consultas, das quais 2480 foram teleconsultas (Quadro I).

Aos doentes em seguimento na Consulta Externa foram disponibilizadas aplicações como SMS, WhatsApp ${ }^{\circledR}$ e endereços eletrónicos para contacto com os médicos do Serviço.

As questões mais frequentemente colocadas pelos doentes estavam relacionadas com o receio de poderem estar infetados, de serem ou não uma população de risco, dúvidas acerca da segurança da sua medicação (particularmente corticosteroides inalados ou tópicos nasais) e agendamentos das administrações de imunoterapia ou terapêutica com biológicos. Outro motivo frequente de contacto foi a renovação de prescrições médicas, facilitado pelo uso de prescrição eletrónica médica (PEM).

\section{Atendimento não programado}

O Serviço de Imunoalergologia disponibiliza aos doentes em seguimento na sua Consulta Externa um serviço de atendimento não programado. Este Serviço destina-se à observação e orientação dos doentes com exacerbação da sua doença de base, apoio às solicitações do serviço de urgência (SU) ou do Internamento dos vários serviços do hospital. Durante este período esta atividade foi restrita à observação de doentes com patologia cutânea, nomeadamente agudizações de urticária e/ou angioedema a necessitarem de tratamento urgente. Todos os doentes que apresentavam doença respiratória em fase de agudização foram encaminhados para o SU, pelo potencial risco de transmissão de SARS-CoV-2. Foi também mantida a resposta às solicitações de doentes internados noutros serviços, onde foram observados, entre outros casos, três doentes com suspeita de drug reaction with eosinophilia and systemic symptoms (DRESS). 


\section{GESTÃO E PLANO DE CONTINGÊNCIA DO SERVIÇO DE IMUNOALERGOLOGIA \\ DO CENTRO HOSPITALAR E UNIVERSITÁRIO DE S. JOÃO \\ NO CONTEXTO DA PANDEMIA POR COVID-19 / PÁGINA EDUCACIONAL}

\section{Imunoterapia}

Os doentes a fazer imunoterapia sublingual (inalantes, látex e Pru $p$ 3) continuaram os seus tratamentos em casa. Os tratamentos iniciais foram adiados.

As administrações de imunoterapia subcutânea com alergénios inalantes realizadas na consulta externa foram suspensas. Os tratamentos iniciais foram adiados e os doentes em fase de manutenção aconselhados a efetuar as administrações programadas nas Unidades de Saúde Familiar/Centros de Saúde na sua área de residência. Disponibilizamos instruções escritas, uma linha telefónica direta para contacto com a equipa de enfermagem da nossa consulta e um endereço eletrónico para esclarecimento e orientação de eventuais dúvidas.

Em relação à imunoterapia com veneno de himenópteros, por se tratar de um tratamento life-saving, consideramos que os tratamentos de manutenção não deveriam ser suspensos durante este período. A decisão da continuidade dos tratamentos foi individualizada, partiIhando com cada doente as informações relativas ao risco/benefício da sua continuação. Relativamente aos tratamentos iniciais, a decisão foi de adiar, face ao risco de reações sistémicas e para evitar uma permanência mais prolongada no Hospital de Dia.

\section{Terapêutica com biológicos}

Os doentes sob terapêutica com biológicos para asma grave (omalizumab, mepolizumab, reslizumab e benralizumab) e urticária (omalizumab) faziam as respetivas administrações no Hospital de Dia, enquanto o dupilumab, utilizado para tratamento da dermatite atópica grave, existia já sob a forma de seringa pré-cheia para autoadministração domiciliária. No entanto, estava em elaboração um projeto para os doentes efetuarem a autoadministração domiciliária de biológicos. A pandemia COVID-19 veio acelerar a implementação deste projeto. As soluções liofilizadas do omalizumab, mepolizumab e benralizumab foram substituídas por dispositivos de autoadministração (seringa pré-cheia ou autoinjetor), foram redigidos protocolos de treino e folhetos informativos para os doentes. Os doentes ou seus cuidadores foram treinados pela equipa de enfermagem. Os doentes a fazer reslizumab, os que não foram capazes de fazer corretamente a autoadministração de biológicos ou que preferiram continuar a fazer o tratamento no hospital mantiveram os seus agendamentos no Hospital de Dia.

\section{Hospital de Dia}

Os procedimentos de diagnóstico relacionados com a alergia a fármacos, alimentos ou veneno de himenópteros são realizados em Hospital de Dia. Estes procedimentos podem ocasionar sintomas digestivos ou respiratórios, com potencial risco de transmissão de SARS-CoV-2. Por este motivo foram suspensos, com exceção daqueles que consideramos como prestação de cuidados essenciais, designadamente a dessensibilização a fármacos antineoplásicos. Durante este período foram realizados 16 destes procedimentos (Quadro I).

\section{Meios complementares de diagnóstico}

O Serviço de Imunoalergologia dispõe de um Laboratório de exploração funcional respiratória localizado no espaço físico da Consulta Externa. Durante este período todos os exames de função respiratória, nomeadamente espirometrias, oscilometrias de impulso, provas de metacolina e doseamentos do óxido nítrico exalado, foram suspensos.

Da mesma forma, a realização de testes cutâneos pela nossa equipa de enfermagem foi, nas suas várias vertentes, suspensa.

\section{EQUIPA MÉDICA}

Durante este período os médicos do Serviço de Imunoalergologia mantiveram os seus turnos de consulta e horários alocados ao serviço de permanência e consulta externa, com exceção dos médicos que tiveram de prestar apoio a filhos menores ou com comorbilidades de risco para COVID-19, que realizaram apenas teletrabalho. 
Foi disponibilizado o acesso remoto às aplicações SClínico ${ }^{\circledR}, \mathrm{PEM}^{\circledR}$ e $\mathrm{JOne}^{\circledR}$ (software clínico desenvolvido pelo CHUSJ) para realização de teleconsultas e outras tarefas assistenciais (como a elaboração de relatórios clínicos, agendamento de consultas ou requisição de meios complementares de diagnóstico) a partir dos seus domicílios.

A comunicação entre os profissionais do Serviço passou a ser quase exclusivamente realizada através de smartphones hospitalares, WhatsApp ${ }^{\circledR}$ e correio eletrónico.

Todos os internos e alguns dos assistentes hospitalares, considerando a gravidade da situação e da redução da atividade clínica programada, voluntariaram-se para trabalhar em diversas atividades relacionadas com a COVID-19, mantendo paralelamente a sua atividade programada no Serviço de Imunoalergologia. Foram integrados em equipas coordenadas pelo Serviço de Doenças Infeciosas, serviço que encabeçou a resposta à COVID-19 no CHUSJ, efetuando o seguimento por teleconsulta de doentes infetados ou com suspeita de infeção, trabalhando nas enfermarias de doentes internados com COVID-19 e realizando turnos de urgência na avaliação de doentes com suspeita de infeção por COVID-19.

O aumento exponencial de doentes infetados levou - CHUSJ a reduzir o número de internamentos por COVID-19, desenvolvendo um home-care program para seguimento de doentes que não apresentavam critérios de doença grave à observação no SU. Um médico telefonava ao doente a informar da positividade do teste rRT-PCR SARS-CoV-2 e a recomendar as necessárias medidas de isolamento. Em função do estado do doente e das suas comorbilidades, utilizando um protocolo definido pelo Serviço de Doenças Infeciosas, a equipa médica efetuava teleconsultas de avaliação a cada 24-72h, orientava quando necessário os doentes para observação no SU, ajustava a terapêutica e referenciava os doentes que necessitavam de suporte psicológico ou de assistência pelos serviços sociais.

Durante este período, a equipa de médicos do Serviço de Imunoalergologia, sob coordenação do Serviço de
Doenças Infeciosas e mais tarde reforçada por alguns médicos dos Serviços de Dermatovenereologia, Anatomia Patológica e Reumatologia, realizou mais de II 000 teleconsultas e acompanhou cerca de 1700 doentes com COVID-19 (Quadro I).

\section{ATIVIDADES FORMATIVAS E INVESTIGAÇÃO}

\section{Reuniões de serviço e internato médico}

As atividades formativas, como as reuniões de serviço nas suas várias modalidades, foram suspensas durante este período. Por decisão superior, as valências do internato médico foram suspensas e adiadas até final do estado de emergência.

O recurso a plataformas digitais, como $\circ \mathrm{Zoom}^{\circledR}$, WhatsApp ${ }^{\circledR}$ e correio eletrónico, ou o uso de smartphones hospitalares, serviram de suporte para a discussão de casos clínicos e sua orientação.

\section{Ações de formação nacionais e internacionais}

Os médicos do serviço tiveram a oportunidade de assistir a numerosas ações de formação ou congressos através de plataformas digitais. Os internos de formação específica foram incentivados a participar ativamente no Congresso Anual da Academia Europeia de Alergia e Imunologia Clínica (EAACl), em formato digital, assistindo às diversas sessões e apresentando trabalhos de investigação.

\section{Investigação}

As atividades de investigação com contactos presenciais foram suspensas. Foram desenvolvidos novos projetos de investigação relativos à COVID-19.

\section{RETOMA DA ATIVIDADE}

A principal limitação à retoma, que se iniciou a 18 de maio, foi a necessidade de evitar a sobrelotação dos es- 


\section{GESTÃO E PLANO DE CONTINGÊNCIA DO SERVIÇO DE IMUNOALERGOLOGIA \\ DO CENTRO HOSPITALAR E UNIVERSITÁRIO DE S. JOÃO \\ NO CONTEXTO DA PANDEMIA POR COVID-19 / PÁGINA EDUCACIONAL}

paços de espera, comuns a outros serviços clínicos hospitalares (Quadro I).

Relativamente às primeiras consultas, foi decidido caso a caso se seriam ou não realizadas presencialmente, de acordo com a informação transmitida no pedido de consulta. Genericamente, eram mantidas em modelo não-presencial as consultas motivadas por suspeita de alergia a fármacos, alergia alimentar ou urticária, recorrendo-se ao envio de informação do doente por via eletrónica sempre que necessário. Para recuperar mais rapidamente a lista de espera para primeira observação, foi inicialmente duplicado o número de primeiras consultas por turno, aproveitando o facto das consultas subsequentes se manterem maioritariamente em formato de teleconsulta. Estas primeiras consultas foram marcadas com intervalos de Ih para evitar a sobrelotação das salas de espera. Em termos de agendamentos, em julho a atividade de consulta voltou praticamente ao funcionamento habitual pré-pandemia.

A consulta de atendimento não programado mantém-se ainda com as restrições já descritas.

As administrações de imunoterapia com aeroalergénios foram reiniciadas com alargamento do tempo de agendamento para cada doente de 15 para 20 minutos, evitando-se a sobrelotação dos espaços de espera. As provas de função respiratória foram reiniciadas mais tarde, com alargamento do tempo entre doentes e reforço das medidas de higienização, e apenas após obtenção de parecer favorável da Unidade de Prevenção e Controlo da Infeção e Resistência aos Antimicrobianos (UPCIRA). Não foi ainda reiniciada a realização de prova de metacolina, por ser um procedimento gerador de aerossóis. A realização de testes cutâneos, nas suas diversas vertentes, foi retomada como habitual.

A atividade em Hospital de Dia foi retomada, tendo-se inicialmente reduzido o número de doentes por sessão, verificando-se posteriormente, que era possível garantir o distanciamento adequado com o número normal de doentes. Foi mantido o programa de autoadministração de biológicos.
De acordo com as instruções da UPCIRA, a atividade clínica tem sido assegurada com recurso a EPI de nível I. Os espaços foram reorganizados de modo a ser respeitado o correto distanciamento entre profissionais e utentes, foi aumentada a frequência da higienização dos mesmos e genericamente interdita a presença de acompanhantes. Os técnicos de diagnóstico e terapêutica realizam as provas de função respiratória com EPI de nível 2. À entrada no centro de ambulatório médico, todos os doentes têm de higienizar as mãos e colocar máscara cirúrgica. A entrada dos doentes no Hospital de Dia é condicionada à avaliação da temperatura corporal e realização de questionário de sintomas e exposição de risco, sendo efetuada pesquisa de SARS-CoV-2 na orofaringe/nasofaringe dos casos suspeitos. A lotação das salas de espera foi reduzida a cerca de $30 \%$.

\section{CONCLUSÕES}

A pandemia COVID-19, bem como a implementação de medidas decretadas pelo governo e DGS, colocaram os prestadores de cuidados de saúde, muito particularmente os hospitais de referência para COVID-19 e os seus serviços clínicos, perante alterações dramáticas e inesperadas relativamente à sua atividade clínica, formativa e de investigação. O Serviço de Imunoalergologia, a par de outros serviços clínicos do CHUSJ, teve de, num curto espaço de tempo, tomar um conjunto de decisões relativas à atividade clínica assistencial. Novos procedimentos tiveram de ser equacionados para garantir a segurança dos profissionais de saúde e dos doentes.

Neste contexto tivemos de elaborar, em menos de 24 horas, um plano de contingência, discriminando as tarefas a desempenhar por cada médico e as ações a tomar em relação às diversas atividades assistenciais realizadas pelo Serviço de Imunoalergologia, garantindo sempre a segurança dos profissionais de saúde e utentes (Quadro I). As nossas decisões foram discutidas em grupo, baseadas na nossa experiência, sentido crítico e 
bom senso clínico, tanto mais que nesta fase inicial não existiam ainda quaisquer recomendações ou guidelines disponíveis. O primeiro artigo a mencionar as decisões que uma unidade de imunoalergologia tomou neste âmbito foi publicado online apenas no final de março ${ }^{6}$. Posteriormente, já em maio, foi publicado por Shaker et al ${ }^{7}$ uma proposta de um plano de contingência para um serviço ou unidade de imunoalergologia. Só muito recentemente foi publicado um position paper da EAACI/ARIA acerca deste tema ${ }^{8}$. Não obstante, as medidas que tomamos logo em março e que serviram de base à elaboração do plano de contingência definido para o nosso serviço, não diferiram no essencial das medidas que foram apresentadas posteriormente nestes artigos ${ }^{6-8}$.

Uma avaliação rigorosa do impacto da COVID-19 na atividade clínica só será possível quando a pandemia for dada como terminada e puderem ser contabilizados os períodos de suspensão da atividade e as estratégias adotadas para os colmatar. As medidas que implementamos permitiram que fosse assegurada uma produção, ao nível das consultas subsequentes, similar a anos anteriores, visto que praticamente não foram desmarcadas (apenas menos 33 consultas que entre março e maio de 2019). Verificou-se uma redução de $322(36,8 \%)$ primeiras consultas face a igual período de 2019. No entanto, é de salientar que as consultas canceladas em março e abril foram recuperadas durante o mês de maio, através da duplicação do número de primeiras consultas realizadas em cada turno. Assim, esta diminuição poderá estar relacionada com uma menor referenciação à especialidade pelos Cuidados de Saúde Primários, que apenas em agosto vão retomar a sua carteira de serviços habitual. $\mathrm{O}$ impacto principal da pandemia verificou-se ao nível dos procedimentos de diagnóstico em Hospital de Dia, visto que nos é muito difícil efetuar agendamentos adicionais destes procedimentos. Paralelamente, a qualidade dos cuidados prestados foi afetada pela impossibilidade de observar presencialmente os doentes durante o período em que vigorou o plano de contingência ou pela diminuição do acesso a meios complementares de diagnóstico.
A gestão da atividade clínica do serviço teve de ser coordenada com a resposta às necessidades levantadas pela COVID-19. Os médicos do serviço participaram ativamente no seguimento por teleconsulta de doentes infetados ou com suspeita de infeção, trabalhando nas enfermarias de doentes internados e no SU. Realizaram trabalho em horário noturno e fins de semana. O seu trabalho e dedicação foram amplamente reconhecidos, com agradecimento público por parte da responsável pela COVID-19 no CHUSJ, da direção clínica e do conselho de administração.

A atividade formativa foi também fortemente comprometida durante as 8 semanas em análise, dado que os estágios dos internos de formação específica foram suspensos. Também neste caso existiu a necessidade de realocar horários de trabalho, e respetivas tarefas, aos internos que se encontravam a fazer as suas valências noutros serviços e que regressaram ao Serviço de Imunoalergologia. Não tendo sido emitidas orientações claras sobre a recuperação deste tempo, eventuais adaptações ao plano de formação serão, por ora, decididas caso a caso, tendo em consideração o momento do programa em que cada interno se encontrava e as atividades que desemprenhou neste período.

As reuniões científicas presenciais foram suspensas. O recurso a plataformas digitais, como $\circ$ Zoom $^{\circledR}$, WhatsApp ${ }^{\circledR}$ e correio eletrónico, ou o uso de smartphones hospitalares, serviram de suporte para a discussão de casos clínicos, participação em numerosas ações de formação, congressos nacionais e internacionais. Apesar do seu forte envolvimento na resposta à COVID-19, os internos de formação especifica participaram ativamente no congresso anual da EAACI.

A pandemia COVID-19 foi inesperada e teve um impacto tremendo na prestação dos cuidados de saúde e nas instituições. O Serviço de Imunoalergologia, tal como outros serviços clínicos do CHUSJ, não tinha definido um plano de ação para emergências deste tipo. Teve de rapidamente reorganizar a sua atividade e elaborar um plano de contingência. As decisões que tomámos foram baseadas na experiência e bom senso clínico dos seus 


\section{GESTÃO E PLANO DE CONTINGÊNCIA DO SERVIÇO DE IMUNOALERGOLOGIA \\ DO CENTRO HOSPITALAR E UNIVERSITÁRIO DE S. JOÃO \\ NO CONTEXTO DA PANDEMIA POR COVID-19 / PÁGINA EDUCACIONAL}

profissionais, dado que à data não estavam disponíveis quaisquer normas ou recomendações.

A gestão da pandemia por COVID-19 teve, apesar de tudo, alguns aspetos positivos na dinâmica do serviço. Antes de mais, reposicionou o papel das plataformas digitais, nomeadamente a teleconsulta, no seguimento dos doentes com patologia alérgica bem controlada. Contribuiu para a implementação de novos projetos, de que são exemplos a disponibilização de aplicações (SMS, WhatsA$\mathrm{PP}^{\circledR}$, endereços eletrónicos) aos utentes para contacto com os médicos, a autoadministração de biológicos e a realização de reuniões e discussão de casos clínicos online.

Por fim, veio estimular o trabalho em equipa, a capacidade organizativa e revelar, de forma muito clara, a enorme disponibilidade, profissionalismo e empenho dos seus colaboradores. Foi com esta atitude que o Serviço de Imunoalergologia do CHUSJ ultrapassou este período de incerteza e dificuldade, assegurando sempre os cuidados aos doentes alérgicos e contribuindo de forma muito relevante para a luta contra a COVID-19.

\section{Conflito de interesses}

Os autores declaram que não existem conflitos de interesse.

\section{AGRADECIMENTOS}

Os autores desejam agradecer o trabalho e dedicação de todos os elementos do Serviço de Imunoalergologia. A forma empenhada e corajosa com que encararam todos os desafios permitiu não só atingir os resultados aqui apresentados, mas também garantir cuidados de saúde de alta qualidade a todos os doentes alérgicos ao nosso cuidado e a centenas de doentes com COVID-19.
Contacto:

Leonor Carneiro-Leão

Serviço de Imunoalergologia

Centro Hospitalar de São João, Porto

Alameda Prof Hernâni Monteiro

4200-319, Porto

Email: leonorcarneiroleao@gmail.com

\section{REFERÊNCIAS}

I. Li Q, Guan X, Wu P, Wang X, Zhou L, Tong Y, et al. Early transmission dynamics in Wuhan, China, of novel coronavirus-infected pneumonia. N Engl J Med 2020;382(I3): I 199-207.

2. Coronavirus disease 2019 (COVID-19): situation report, 132. World Health Organization. (2020). Available at https://www. who.int/emergencies/diseases/novel-coronavirus-2019/situation -reports. Accessed May 31, 2020.

3. Zhang JJ, Dong X, Cao YY, Yuan YD, Yang YB, Yan YQ, et al. Clinical characteristics of 140 patients infected with SARS-CoV-2 in Wuhan, China. Allergy 2020;75(7):1730-4I.

4. Coronavirus disease 2019 (COVID-19): situation report, 061. Portuguese Directorate-General of Health: Lisbon; 2020. Available at https://www.dgs.pt/em-destaque/relatorio-de-situacao -n-06I-02052020.aspx. Accessed May 3I, 2020.

5. European Centre for Disease Prevention and Control. Infection prevention and control for COVID-19 in healthcare settings March 2020. ECDC: Stockholm; 2020.

6. Codispoti CD, Bandi S, Moy JN, Mahdavinia M. Running a virtual allergy 134 division and training program in the time of COVID-19 pandemic. J Allergy Clin Immunol 2020;145(5):I357-9.

7. Pfaar O, Klimek L, Jutel M, Akdis CA, Bousquet J, Breitenede $H$, et al. COVID-19 pandemic: Practical considerations on the organization of an allergy clinic - an EAACI/ARIA Position Paper [published online ahead of print, 2020 Jun 12]. Allergy 2020; 10. IIII/ all. 14453.

8. Shaker MS, Oppenheimer J, Grayson M, Stukus D, Hartog N, Hsieh EWY, et al. COVID-19: Pandemic contingency planning for the allergy and immunology clinic. J Allergy Clin Immunol Pract 2020;8(5):1477-88.e5. 